\title{
Prevalência de anemia e correlação da concentração de hemoglobina com fatores cognitivos em idosos
}

\author{
Prevalence of anemia and correlation between the concentration \\ of hemoglobin and cognitive factors among the elderly
}

Natane Daiana Silva Sousa ${ }^{1}$

Tarciana Nobre de Menezes ${ }^{1}$

Nathalie de Almeida Silva ${ }^{1}$

Maria do Carmo Eulálio ${ }^{2}$

Adriana de Azevedo Paiva ${ }^{3}$

${ }^{1}$ Programa de PósGraduação em Saúde Pública, Universidade Estadual da Paraíba (UEPB). Av. Baraúnas 351, Bodocongó. 58109-753

Campina Grande PB Brasil. natane.sousa@hotmail.com ${ }^{2}$ Departamento de Psicologia, Centro de Ciências Biológicas e da Saúde, UEPB. Campina Grande PB Brasil.

${ }^{3}$ Departamento de Nutrição, Centro de Ciências da

Saúde, Universidade Federal do Piauí. Teresina PI Brasil.
Abstract The scope of this study was to determine the prevalence of anemia and the correlation between the concentration of hemoglobin and cognitive factors in an elderly population group resident in Campina Grande, Paraiba, Brazil. It was a cross-sectional study with individuals aged 60 or older. Men with hemoglobin levels $<13$ $g / d L$ and women with levels $<12 \mathrm{~g} / \mathrm{dL}$ were considered anemic. Sociodemographic, cognitive condition and nutritional status variables were analyzed. Statistical analysis was performed by means of simple and multiple linear regression. Among the 360 elderly patients evaluated (67.2\% women), the prevalence of anemia was $12.5 \%$. The average concentration of hemoglobin found was $13.5 \mathrm{~g} / \mathrm{dL}$ and was correlated to sex variables $(\beta=-0.44,95 \%$ CI: $-1.35,-0.85)$, age $(\beta=-0.14$ ; 95\% CI: $-0.03,-0.01)$, nutritional status $(\beta=$ 0.16; 95\% CI: 0.01, 0.06), memory impairment $(\beta=0.12 ; 95 \% C I:-0.06,-0.01)$, and dementia ( $\beta=-0.13,95 \%$ CI: -0.06, -0.01). Routine blood testing among the elderly makes it possible to detect and treat anemia at an early stage. Actions geared towards the elderly who show a cognitive decline should be developed in the Basic Family Health Units in order to improve the quality of life of this population.

Key words Anemia, Hemoglobin, Risk factors, Cognition, Elderly
Resumo $O$ objetivo deste estudo foi verificar a prevalência de anemia e a correlação da concentração de hemoglobina com fatores cognitivos em população idosa residente em Campina Grande, Paraíba, Brasil. Tratou-se de um estudo transversal com indivíduos de 60 anos de idade ou mais. Foram considerados anêmicos os indivíduos do sexo masculino que apresentaram concentração de hemoglobina $<13 \mathrm{~g} / \mathrm{dL}$ e do feminino que apresentaram concentrações $<12 \mathrm{~g} / \mathrm{dL}$. Foram analisadas variáveis sociodemográficas, condição cognitiva e estado nutricional. A análise estatística foi realizada por meio da regressão linear simples e múltipla. Foram avaliados 360 idosos (67,2\% mulheres), cuja prevalência de anemia foi de $12,5 \%$. A concentração média de hemoglobina verificada foi de 13,5 g/dL e esteve correlaciona$d a$ às variáveis sexo $(\beta=-0,44$; IC95\%: $-1,35$;$0,85)$, idade ( $\beta=-0,14 ;$ IC95\%: $-0,03 ;-0,01)$, estado nutricional ( $\beta=0,16$; IC95\%: 0,01;0,06), comprometimento de memória $(\beta=0,12 ;$ IC95\%: -0,06;-0,01), e demência ( $\beta=-0,13 ;$ IC95\%: -0,06;-0,01). A realização rotineira do hemograma dos idosos proporciona identificar e tratar precocemente a anemia. Ações voltadas para os idosos que apresentam declinio cognitivo devem ser desenvolvidas nas Unidades Básicas de Saúde da Família, a fim de melhorar a qualidade de vida desta população.

Palavras-chave Anemia, Hemoglobina, Fatores de risco, Cognição, Idoso 


\section{Introdução}

A anemia constitui um distúrbio hematológico relevante, caracterizado pela redução da concentração de hemoglobina no sangue ${ }^{1}$, o qual tem se destacado na população idosa, por ser uma condição de elevada prevalência neste grupo etário². Pesquisas sobre anemia em idosos têm sido realizadas nos últimos anos, tanto no Brasil ${ }^{3-5}$ como internacionalmente ${ }^{6-9}$, ressaltando a relevância do estudo acerca desta doença no contexto mundial.

As alterações fisiológicas, funcionais e bioquímicas que ocorrem no corpo humano com o processo de envelhecimento acarretam uma tendência de redução da concentração de hemoglobina $^{10-12}$. No entanto, essa condição não deve ser considerada consequência natural do envelhecimento ${ }^{13}$, uma vez que a anemia repercute negativamente na saúde dos idosos, agravando doenças já existentes e contribuindo para o surgimento de novas complicações ${ }^{4,14}$, além de gerar impactos consideráveis de custos para o sistema de saúde, tendo em vista que a doença está associada à maior utilização dos serviços de saúde ${ }^{15}$ e maior permanência no ambiente hospitalar ${ }^{16}$.

$\mathrm{Na}$ literatura, a anemia e as baixas concentrações de hemoglobina em idosos residentes na comunidade têm sido associadas ao declínio $\operatorname{cognitivo~}^{17,18}$, como demência, depressão e comprometimento de memória, maior risco para o desenvolvimento da Doença de Alzheimer ${ }^{19,20}$, pior estado nutricional ${ }^{4,21}$ e maior risco de mortalidade $^{22-24}$.

Os instrumentos comumente utilizados para a avaliação cognitiva em idosos são o Mini Exame do Estado Mental ${ }^{25-27}$, a Escala de Depressão Geriátrica $^{28-30}$ e o Questionário de Medidas de Queixas Subjetivas de Memória ${ }^{17,31}$. Estudo de base populacional realizado, na Itália, com 717 idosos, utilizando para a avaliação do declínio cognitivo, dentre outros instrumentos o Mini Exame do Estado Mental e a Escala de Depressão Geriátrica, verificou associação entre a anemia e o declínio cognitivo ${ }^{29}$.

Como visto, o declínio cognitivo associado à baixa concentração de hemoglobina pode comprometer a qualidade de vida da pessoa idosa, interferindo na realização das atividades cotidianas e na interação social ${ }^{29,32}$. O presente estudo teve por objetivo verificar a prevalência de anemia e a correlação da concentração de hemoglobina com fatores cognitivos em população idosa residente em Campina Grande, Paraíba, Brasil.

\section{Métodos}

Tratou-se de um estudo do tipo transversal com coleta de dados primários. A população foi constituída por indivíduos com 60 anos ou mais, de ambos os sexos, cadastrados na Estratégia Saúde da Família do município de Campina Grande/PB.

Para o cálculo da amostra, estimou-se uma prevalência dos desfechos de, no mínimo, 25\%. $\mathrm{O}$ cálculo do tamanho amostral foi realizado a partir da seguinte equação: $\left\{\left[E^{2} \times \mathrm{p}(1-\mathrm{p})\right] \times \mathrm{c}\right\} /$ $A^{2}$. Onde $E$ é o limite de confiança $(1,96), c$ é o coeficiente de correlação amostral $(2,1)$, considerando o procedimento por conglomerado, e $A$ é a precisão aceita (6\%) para a prevalência estimada. A amostra foi proporcional a cada um dos seis Distritos Sanitários de Campina Grande, sendo composta por 420 idosos.

A seleção da amostra considerou os seis distritos sanitários do município, sendo sorteada uma Unidade Básica de Saúde da Família (UBSF) em cada um deles. Após a seleção das UBSF, realizou-se um levantamento do número de idosos nelas cadastrados. A partir daí foi calculada a proporção de idosos por unidade, em relação ao total das seis unidades. Tal proporcionalidade definiu o número de idosos amostrais por UBSF. Sendo assim, nas seis UBSF sorteadas foram entrevistadas as seguintes proporções (números) de idosos: 9,6\% (40), 11,4\% (48), 14,5\% (61), $8,6 \%$ (36), 43,3\% (182) e 12,6\% (53), totalizando os 420 idosos da amostra. Em cada UBSF procedeu-se ao sorteio sistemático dos idosos com a elaboração de uma lista com os nomes de todos os idosos cadastrados. O número a ser saltado, até chegar ao próximo idoso da lista a ser entrevistado, foi definido a partir da razão entre o número total dos idosos cadastrados e o número dos idosos determinados para serem entrevistados naquela UBSF, gerando assim o número cinco. Como o cálculo foi proporcional, então o número de saltos na lista foi o mesmo em todas as UBSF. Dessa forma, a cada idoso selecionado, foram saltados quatro idosos da lista. $\mathrm{O} 5^{\circ}$ idoso foi o selecionado, e assim sucessivamente, a fim de se obter melhor distribuição e garantia de que toda a lista fosse percorrida.

Foram excluídos do estudo os idosos em fase terminal de doença, ou seja, aqueles sem possibilidades terapêuticas e que apresentavam debilidade clínica grave; e os que estavam ausentes do município durante o tempo da pesquisa de campo na área de abrangência da Estratégia Saúde da Família em que eram cadastrados. 
No primeiro momento foi realizada a entrevista no domicílio do idoso, na qual foram coletadas as informações sociodemográficas, cognitivas, e do estado nutricional. Nesse momento, o idoso foi orientado a comparecer em jejum de 12 horas, em data e horário determinados, à UBSF de abrangência para a coleta do sangue e posterior análise da hemoglobina sérica.

A coleta do sangue foi realizada por profissionais habilitados e as análises foram realizadas em um Laboratório de Análises Clínicas, devidamente credenciado e cadastrado no Serviço de Controle e Qualidade da Sociedade Brasileira de Análises Clínicas. Para reduzir as perdas de indivíduos na coleta de sangue, estabeleceu-se contato com os idosos que faltaram à primeira coleta, sendo marcada nova data para realização da coleta de sangue. Após a segunda falta, foi estabelecido novo contato e a coleta de sangue foi remarcada para ser realizada no domicílio do idoso. Em caso de nova recusa, esse indivíduo era considerado como perdido para os dados referentes às análises bioquímicas.

As variáveis deste estudo foram a concentração de hemoglobina $(\mathrm{Hb})(\mathrm{g} / \mathrm{dL})$ e as cognitivas (demência, depressão e comprometimento de memória). Foram consideradas variáveis de ajuste as sociodemográficas (sexo, idade e anos de estudo) e o estado nutricional.

A prevalência de anemia foi estimada por meio da concentração de hemoglobina $(\mathrm{Hb})(\mathrm{g} /$ $\mathrm{dL}$ ), a qual foi determinada por Automação, ABX PENTRA 80. Foram considerados com anemia, indivíduos do sexo masculino que apresentaram concentração de hemoglobina $<13 \mathrm{~g} / \mathrm{dL}$ e do sexo feminino que apresentaram concentração $<$ $12 \mathrm{~g} / \mathrm{dL}^{33}$.

As variáveis cognitivas foram tratadas de forma contínua, por meio do número de pontos obtidos no Mini-Exame do Estado Mental $(\mathrm{MEEM})^{25,34}$, o qual avaliou a demência, na Escala de Depressão Geriátrica (EDG) ${ }^{28,35}$ que avaliou a depressão e no Questionário de Medidas de Queixas Subjetivas de Memória (MAC-Q) ${ }^{31}$, o qual avaliou o comprometimento de memória.

O Mini Exame do Estado Mental (MEEM) é composto por questões que podem ser agrupadas em 7 categorias cognitivas: orientação temporal
(5 pontos), orientação espacial (5 pontos), registro de 3 palavras ( 3 pontos), atenção e cálculo ( 5 pontos), memória (3 pontos), linguagem (8 pontos) e capacidade construtiva visual (1 ponto). Este instrumento resulta em um escore que pode variar de 0 até um total máximo de 30 pontos, no qual o número de pontos e o prejuízo cognitivo são inversamente proporcionais. A Escala de Depressão Geriátrica (EDG) é composta por 15 perguntas, cada uma com duas alternativas de respostas: Sim e Não, com valores atribuídos de 0 a 1 ponto, e o escore é dado pelo somatório dos pontos obtidos, quanto maior a pontuação, maior o número de sintomas depressivos presentes. O Questionário de medida de queixas subjetivas de memória - Subjective Perception of Memory Complaints Questionnaire (MAC-Q) - é um breve questionário utilizado a fim de identificar o declínio da memória em idosos. O MAC-Q é composto por 6 itens, avaliados segundo uma escala Likert, variando de muito melhor agora a muito pior agora. O escore mínimo é de 7 pontos e quanto maior a pontuação, maior é a percepção de queixas de memória.

Quanto à idade, o idoso foi categorizado como pertencente ao grupo etário de 60 a 69 anos, de 70 a 79 anos, ou de 80 anos ou mais. No que se refere à variável anos de estudo, o idoso foi classificado como analfabeto, apresentando de 1 a 4 anos de estudo, de 5 a 8 anos de estudo, de 9 a 11 anos de estudo ou de 12 anos ou mais de estudo. A avaliação do estado nutricional foi realizada por meio do Índice de Massa Corporal (IMC), para o qual foram mensurados a estatura e o peso, com base nas técnicas propostas por Gordon et al. ${ }^{36}$. Para análise do IMC $\left(\mathrm{kg} / \mathrm{m}^{2}\right)$ foi utilizada a classificação sugerida pela Organização Pan-Americana de Saúde ${ }^{37}$ (OPAS):baixo peso ( $<23)$, eutrofia $(\geq 23 \mathrm{e}<28)$, sobrepeso $(\geq$ 28 e $<30)$ e obesidade $(\geq 30)$.

\section{Análise Estatística}

O banco de dados foi elaborado utilizandose o aplicativo Microsoft Office Excel. As informações estatísticas foram obtidas com o auxílio do aplicativo estatístico SPSS 22.0 (IBM Corp., Armonk, Estados Unidos). Utilizou-se o teste de Kolmogorov-Smirnov para verificar a normalidade na distribuição da concentração de hemoglobina.

Os dados foram estudados com o intuito de analisar a prevalência de anemia e correlação da concentração de hemoglobina com fatores cognitivos em idosos residentes no município de 
Campina Grande, Paraíba. Para a análise de correlação, utilizou-se o coeficiente de correlação de Pearson e regressão linear simples. Para o modelo de regressão linear múltipla foram consideradas as variáveis que apresentaram valor de $\mathrm{p}<0,20$, obtido na análise bivariada. Considerou-se o método de entrada stepwise forward para cálculo do coeficiente de determinação ajustado $\left(\mathrm{R}_{\mathrm{aj}}^{2}\right)$, com intervalo de confiança de 95\% (IC95\%). Foram verificados, ainda, os resíduos, por meio do teste de multicolinearidade (VIF), e a homoscedasticidade do modelo.

No modelo final, foram consideradas aquelas variáveis que apresentaram correlação estatisticamente significativa com a concentração de hemoglobina $(\mathrm{p}<0,05)$. No entanto, podem ter sido incluídas outras variáveis, independente da significância estatística (variáveis de ajuste).

\section{Considerações éticas}

O estudo maior do qual esta pesquisa faz parte foi aprovado pelo Comitê de Ética e Pesquisa da Universidade Estadual da Paraíba e encontra-se em concordância com os princípios éticos contidos na Declaração de Helsinque da Associação Médica Mundial. Todos os idosos assinaram o Termo de Consentimento Livre e Esclarecido depois de receberem explicações verbais e escritas a respeito do estudo.

\section{Resultados}

Dentre os 420 idosos selecionados para compor a amostra, não foi possível realizar a coleta sanguínea de 60 idosos, os quais foram excluídos da pesquisa. Sendo assim, a amostra deste estudo contou com 360 idosos (67,2\% do sexo feminino) cuja idade variou de 60 a 104 anos, com média de 71,28 anos $(D P=8,93)$. A prevalência de anemia verificada foi de $12,5 \%$.

Na Tabela 1 é apresentada a distribuição dos idosos de acordo com a presença de anemia e as características sociodemográficas, condição cognitiva e estado nutricional. Observa-se nesta tabela que as variáveis sexo e estado nutricional apresentaram associação estatisticamente significativa com a anemia entre os idosos estudados.

Na Tabela 2 são apresentados os valores referentes à média, desvio-padrão, valor mínimo e valor máximo da concentração de hemoglobina, da idade, do Índice de Massa Corporal (IMC), da pontuação do Mini Exame do Estado Mental (MEEM), da Escala de Depressão Geriátrica
(EDG) e do Questionário de Medidas de Queixas Subjetivas de Memória (MAC-Q). Por meio desta tabela, observa-se que a concentração média de hemoglobina verificada entre os idosos foi de $13,5 \mathrm{~g} / \mathrm{dL}$.

Na Tabela 3 são apresentados os resultados da análise de regressão linear simples entre a concentração de hemoglobina e as variáveis independentes: demência (Mini Exame do Estado Mental), depressão (Escala de Depressão Geriátrica), comprometimento de memória (Questionário de Medidas de Queixas Subjetivas de Memória), idade, estado nutricional (Índice de Massa Corporal) e sexo. É possível observar que houve correlação significativa entre a concentração de hemoglobina e o comprometimento de memória $\left(R^{2}=0,02 ; p=0,002\right)$, a idade $\left(R^{2}\right.$ $=0,02 ; \mathrm{p}=0,014)$ e o sexo $\left(\mathrm{R}^{2}=0,15 ; \mathrm{p}<0,001\right)$.

Na Tabela 4 são apresentados os resultados da regressão linear múltipla entre a concentração de hemoglobina e as variáveis independentes. Para a elaboração deste modelo final, foram testados modelos com a variável demência (MEEM) e com a depressão (EDG), independentemente dos valores de significância obtidos na análise bivariada, pois essas variáveis são de grande interesse para o estudo. Além disso, foi considerada a inclusão da variável anos de estudo, a qual poderia exercer influência sobre as outras variáveis do estudo.

As variáveis que compõem o modelo final explicaram $22 \%$ da variação da concentração de hemoglobina entre os idosos. O sexo, a idade, o estado nutricional, o comprometimento de memória e a demência foram as variáveis preditivas $(\mathrm{p}<0,05)$ para alteração da concentração de hemoglobina em idosos. As variáveis anos de estudo e depressão foram consideradas variáveis de ajuste. Apenas o estado nutricional apresentou correlação significativa positiva $(=0,16) \mathrm{com}$ a concentração de hemoglobina, enquanto que a idade, a demência e o comprometimento de memória apresentaram correlação significativa negativa em relação à concentração de hemoglobina. Quanto ao sexo, as mulheres apresentaram maior valor médio de concentração de hemoglobina quando comparadas aos homens.

\section{Discussão}

A prevalência de anemia entre os idosos desta pesquisa foi de $12,5 \%$. Prevalências semelhantes foram encontradas em outros estudos com idosos em diferentes regiões brasileiras, as quais varia- 
Tabela 1. Prevalência de anemia em idosos cadastrados na Estratégia Saúde da Família, segundo características sociodemográficas, condição cognitiva e estado nutricional. Campina Grande, Paraíba, Brasil, 2010.

\begin{tabular}{|c|c|c|c|c|c|c|c|}
\hline \multirow{3}{*}{ Variáveis } & \multicolumn{6}{|c|}{ Anemia } & \multirow{3}{*}{$\begin{array}{l}\text { Valor } \\
\text { de } \mathbf{p}^{*}\end{array}$} \\
\hline & \multicolumn{3}{|c|}{ Sim } & \multicolumn{3}{|c|}{ Não } & \\
\hline & $\mathbf{n}$ & $\%$ & IC95\% & $\mathbf{n}$ & $\%$ & IC95\% & \\
\hline Sexo & & & & & & & 0,014 \\
\hline Masculino & 22 & 18,6 & $(33,7-64,2)$ & 96 & 81,4 & $(25,5-35,9)$ & \\
\hline Feminino & 23 & 9,5 & $(35,8-66,3)$ & 219 & 90,5 & $(64,1-74,6)$ & \\
\hline Grupo etário & & & & & & & 0,328 \\
\hline 60 a 69 anos & 19 & 10,5 & $(27,7-57,8)$ & 162 & 89,5 & $(45,8-57,1)$ & \\
\hline 70 a 79 anos & 15 & 12,8 & $(20,0-49,0)$ & 102 & 87,2 & $(27,3-37,9)$ & \\
\hline$\geq 80$ anos & 11 & 17,7 & $(12,9-39,5)$ & 51 & 82,3 & $(12,4-20,8)$ & \\
\hline Depressão & & & & & & & 1,000 \\
\hline Sim & 12 & 12,5 & $(14,6-41,9)$ & 84 & 87,5 & $(21,9-32,0)$ & \\
\hline Não & 33 & 12,5 & $(58,1-85,4)$ & 231 & 87,5 & $(68,1-78,1)$ & \\
\hline Demência & & & & & & & 0,236 \\
\hline Sim & 33 & 11,5 & $(58,1-85,4)$ & 254 & 88,5 & $(76,1-85,1)$ & \\
\hline Não & 12 & 16,7 & $(14,6-41,9)$ & 60 & 83,3 & $(15,0-24,0)$ & \\
\hline Comprometimento de memória & & & & & & & 0,086 \\
\hline Sim & 37 & 14,4 & $(67,9-92,0)$ & 220 & 85,6 & $(64,4-74,9)$ & \\
\hline Não & 8 & 7,8 & $(8,0-32,1)$ & 95 & 92,2 & $(25,2-35,6)$ & \\
\hline Estado nutricional & & & & & & & 0,024 \\
\hline Eutrofia & 20 & 15,5 & $(32,9-64,9)$ & 109 & 84,5 & $(31,1-42,3)$ & \\
\hline Baixo peso & 11 & 18,0 & $(14,2-42,9)$ & 50 & 82,0 & $(12,7-21,5)$ & \\
\hline Sobrepeso/obesidade & 10 & 6,7 & $(12,4-40,3)$ & 139 & 93,3 & $(40,9-52,5)$ & \\
\hline
\end{tabular}

"Teste qui-quadrado; IC95\%: intervalo de 95\% de confiança.

Tabela 2. Média, desvio-padrão, valor mínimo e valor máximo da concentração de hemoglobina, do Índice de Massa Corporal, da pontuação do Mini Exame do Estado Mental, da Escala de Depressão Geriátrica e do Questionário de Medidas de Queixas Subjetivas de Memória dos idosos. Campina Grande, Paraíba, Brasil, 2010.

\begin{tabular}{lcrrrrr}
\hline Dados Descritivos & $\begin{array}{c}\text { Hemoglobina } \\
(\mathbf{g} / \mathbf{d L})\end{array}$ & $\begin{array}{c}\text { Idade } \\
(\mathbf{a n o s})\end{array}$ & IMC $\left(\mathbf{K g} / \mathbf{m}^{2}\right)$ & $\begin{array}{c}\text { MEEM } \\
\text { (pontos) }\end{array}$ & $\begin{array}{c}\text { EDG } \\
\text { (pontos) }\end{array}$ & $\begin{array}{c}\text { MAC-Q } \\
\text { (pontos) }\end{array}$ \\
\hline Média & 13,52 & 71,28 & 27,44 & 19,99 & 4,30 & 26,57 \\
Desvio-padrão & 1,16 & 8,93 & 4,86 & 4,96 & 2,64 & 4,25 \\
Valor mínimo & 10,60 & 60 & 14,96 & 3 & 0 & 10 \\
Valor máximo & 17,30 & 104 & 42,81 & 29 & 13 & 35 \\
\hline
\end{tabular}

IMC: Índice de Massa Corporal; MEEM: Mini Exame do Estado Mental; EDG: Escala de Depressão Geriátrica; MAC-Q:

Questionário de Medidas de Queixas Subjetivas de Memória.

Tabela 3. Regressão linear simples entre a concentração de hemoglobina e as variáveis independentes em idosos. Campina Grande, Paraíba, Brasil, 2010.

\begin{tabular}{lcccc}
\hline \multicolumn{1}{c}{ Variáveis } & $\mathbf{R}^{\mathbf{2}}$ & $\beta$ & IC95\% & valor de $\mathbf{p}$ \\
\hline MEEM & 0,01 & 0,05 & $-0,14 ; 0,03$ & 0,348 \\
EDG & 0,01 & $-0,04$ & $-0,06 ; 0,02$ & 0,372 \\
MAC-Q & 0,02 & $-0,16$ & $-0,07 ;-0,01$ & $\mathbf{0 , 0 0 2}$ \\
Idade & 0,02 & $-0,11$ & $-0,03 ;-0,01$ & $\mathbf{0 , 0 1 4}$ \\
IMC & 0,01 & 0,10 & $-0,01 ; 0,05$ & 0,055 \\
Sexo & 0,15 & $-0,38$ & $-1,20 ;-0,72$ & $<\mathbf{0 , 0 0 1}$ \\
\hline
\end{tabular}

MEEM: Mini Exame do Estado Mental; EDG: Escala de Depressão Geriátrica; MAC-Q: Questionário de Medida de Queixas Subjetivas de Memória; IMC: Índice de Massa Corporal; $R^{2}$ : Coeficiente de determinação; ; Coeficiente de correlação; IC95\%: Intervalo de 95\% de confiança. 
Tabela 4. Análise de regressão linear múltipla para estimar a predição das variáveis independentes sobre a concentração de hemoglobina em idosos. Campina Grande, Paraíba, Brasil, 2010.

\begin{tabular}{lcccc}
\hline \multicolumn{1}{c}{ Variáveis } & $\mathbf{R}^{2}$ & $\beta$ & IC95\% & valor de $\mathbf{p}$ \\
\hline Sexo & 0,22 & $-0,44$ & $-1,35 ;-0,85$ & $<\mathbf{0 , 0 0 1}$ \\
MAC-Q & & $-0,12$ & $-0,06 ;-0,01$ & $\mathbf{0 , 0 2 3}$ \\
Idade & $-0,14$ & $-0,03 ;-0,01$ & $\mathbf{0 , 0 0 6}$ \\
IMC & & 0,16 & 0,$01 ; 0,06$ & $\mathbf{0 , 0 0 2}$ \\
EDG & & $-0,04$ & $-0,06 ;-0,0106$ & 0,388 \\
MEEM & $-0,13$ & $-0,11 ; 0,14$ & $\mathbf{0 , 0 1 0}$ \\
Anos de estudo & 0,01 & 0,774 \\
\hline
\end{tabular}

MEEM: Mini Exame do Estado Mental; EDG: Escala de Depressão Geriátrica; MAC-Q: Questionário de Medida de Queixas Subjetivas de Memória; IMC: Índice de Massa Corporal; R²: Coeficiente de determinação; : Coeficiente de correlação; IC95\%: Intervalo de $95 \%$ de confiança.

ram de $11 \%$ a $12,8 \% \%^{3,5,11}$. As prevalências de anemia em idosos verificadas nestes estudos realizados no Brasil, bem como a prevalência verificada no presente estudo, apesar de serem inferiores às prevalências encontradas em estudos envolvendo outros grupos etários como crianças ${ }^{38,39}$ e gestantes ${ }^{40,41}$, devem ser consideradas relevantes, devido às repercussões negativas que esta doença gera sobre a saúde e qualidade de vida do idoso, como o aumento do risco de morbidade e mortalidade.

Neste estudo, a concentração média de hemoglobina verificada entre os idosos foi de 13,5 $\mathrm{g} / \mathrm{dL}$, resultado semelhante ao encontrado em pesquisa com idosos residentes na comunidade em Chicago, Estados Unidos, em que a concentração média de hemoglobina foi de $13,3 \mathrm{~g} / \mathrm{dL}^{17}$. Estudo realizado no Brasil com idosos da comunidade participantes da linha de base de coorte de Bambuí, Minas Gerais, verificou que a concentração média de hemoglobina foi igual a 14,5 $\mathrm{g} / \mathrm{dL}^{4}$, resultado superior ao encontrado na presente pesquisa, o que pode ser atribuído ao fato de os idosos que fizeram parte do estudo serem participantes de uma coorte que é desenvolvida na cidade desde 1997, o que pode favorecer a condição de saúde desses idosos, por meio do acompanhamento da saúde dessa população e melhor controle de doenças.

O sexo esteve correlacionado com a concentração de hemoglobina. Estudos apontam que idosos do sexo masculino apresentam níveis de hemoglobina inferiores ao feminino ${ }^{6,42}$, o que pode ser devido, principalmente, à redução na produção de testosterona com o avançar da idade, a qual tem impacto significativo na redução dos níveis de hemoglobina no organismo ${ }^{6}$. Essa correlação entre o sexo e a concentração de hemoglobina em idosos, reforça a necessidade de maior atenção às demandas e necessidades em saúde apresentadas pelos homens ${ }^{43,44}$.

A idade esteve correlacionada negativamente com a concentração de hemoglobina, o que está em conformidade com a literatura, pois estudos apontam que há uma tendência de redução da concentração de hemoglobina em idosos com o avançar da idade ${ }^{12,24,45}$. Estudo longitudinal norte-americano com adultos não anêmicos residentes na comunidade mostrou que ocorre em média uma queda de $0,06 \mathrm{~g} / \mathrm{dL}$ de hemoglobina por $\mathrm{ano}^{46}$. Alguns fatores são apontados a fim de explicar a diminuição da concentração de hemoglobina em idosos, como a capacidade diminuída do rim em produzir o hormônio eritropoetina ${ }^{3} \mathrm{e}$ o aumento das citocinas pró-inflamatórias associadas ao processo de envelhecimento que interferem na resposta das células eritróides à eritropoetina $^{47}$.

Neste estudo, o Índice de Massa Corporal esteve correlacionado positivamente com a concentração de hemoglobina entre os idosos, esta relação pode ser atribuída ao fato de que determinados fatores que acompanham o envelhecimento, além da presença de doenças crônicas e debilidades físicas associadas à idade ${ }^{48}$ podem favorecer a desnutrição entre os idosos ${ }^{49,50}$. Indivíduos desnutridos, normalmente apresentam alimentação insuficiente em ferro, ácido fólico e vitamina $\mathrm{B}_{12}^{51}$, o que leva a baixas concentrações de hemoglobina no organismo, uma vez que esses nutrientes são essenciais para a eritropoiese $\mathrm{e}^{52}$.

De acordo com pesquisas realizadas com idosos residentes na comunidade, baixas concentrações de hemoglobina têm sido associadas com o declínio cognitivo ${ }^{18-20,53}$. Estudo de base populacional envolvendo 793 idosos participantes da Rush Memory and Aging Project, em Chicago, Es- 
tados Unidos, relatou que baixas concentrações de hemoglobina estiveram associadas a piores desempenhos nos testes aplicados a fim de avaliar a função cognitiva global dos idosos, particularmente com relação à memória ${ }^{17}$.

Neste estudo foi verificada correlação negativa entre a concentração de hemoglobina e a pontuação obtida no MAC-Q, desta forma quanto menor foi a concentração de hemoglobina, maior foi a pontuação no MAC-Q, caracterizando maior comprometimento de memória. Essa correlação pode estar relacionada ao fato de idosos com comprometimento de memória apresentam um processo de neuroinflamação, no qual células especializadas do cérebro liberam no organismo moléculas pró-inflamatórias, como as interleucinas e prostaglandinas ${ }^{54,55}$, as quais têm efeito inibidor sobre a eritropoiese ${ }^{11}$.

Este resultado é preocupante, tendo em vista que tanto as baixas concentrações de hemoglobina como o comprometimento de memória na pessoa idosa estão relacionados com o aumento do risco para o desenvolvimento da Doença de Alzheimer $(\mathrm{DA})^{20,56}$, a qual pode levar à perda da independência e autonomia, interferindo negativamente na qualidade de vida do idoso e de sua família. Essa relação parece ser devido ao efeito protetor sobre os tecidos neurais que a eritropoetina apresenta contra o estresse oxidativo, o qual está envolvido na fisiopatologia da $\mathrm{DA}^{57}$. Sendo assim, os baixos níveis de hemoglobina e o comprometimento de memória devem ser identificados e tratados adequadamente, uma vez que esses agravos ocasionam desfechos adversos à saúde dos idosos.

$\mathrm{Na}$ presente pesquisa houve correlação negativa entre a concentração de hemoglobina e a pontuação obtida no Mini Exame do Estado Mental (MEEM), uma vez que quanto menor a concentração de hemoglobina, maior foi a pontuação obtida no Mini Exame do Estado Mental (MEEM), o que representa menor prejuízo cognitivo aos idosos. Este resultado vai de encontro aos de outros estudos, os quais indicaram que baixas concentrações de hemoglobina estiveram associadas a piores desempenhos no MEEM por parte dos idosos, ou seja, menores pontuações ${ }^{20,58,59}$. Estudos apontam que a anemia e a demência são doenças comumente encontradas em idosos, estando concomitantemente presentes em muitos $\operatorname{casos}^{42,60}$, uma das razões indicadas para isso baseia-se no fato de que pacientes com diagnóstico de demência podem estar sujeitos a apresentar alimentação inadequada com maior frequência ${ }^{60}$ e que as deficiências nutricionais são reconhecidamente um fator etiológico importante para a anemia ${ }^{17}$.

Apesar de o delineamento deste estudo ser transversal, não possibilitando, assim, estabelecer uma relação de causa e efeito, o mesmo atende aos objetivos propostos. Ainda que não seja possível extrapolar os resultados deste estudo para a população idosa em geral, há que se considerar a importância da realização de pesquisas com os idosos cadastrados na Estratégia Saúde da Família, por constituírem um grupo representativo dos idosos brasileiros, tendo em vista que esta Estratégia prioritária da Atenção Básica cobre cerca de $64 \%$ da população do Brasil ${ }^{61}$.

Além disso, há que se considerar a relevância da presente pesquisa, pois é um estudo pioneiro no país sobre a correlação da concentração de hemoglobina com fatores cognitivos abrangendo uma população de idosos cadastrados na Estratégia Saúde da Família de um município do Nordeste brasileiro. Observa-se a necessidade de realização de outros estudos sobre a concentração de hemoglobina em diferentes localidades e populações de idosos, uma vez que a anemia e as baixas concentrações de hemoglobina aumentam o risco de morbimortalidade e os custos para os serviços de saúde, além de estarem relacionados com o declínio cognitivo, o que repercute negativamente na saúde e na qualidade de vida dos idosos.

Tendo em vista que a população deste estudo é assistida pela Estratégia Saúde da Família, a realização rotineira do hemograma dos idosos proporciona identificar e tratar precocemente a anemia. Faz-se necessário que o acompanhamento da saúde dos idosos nas Unidades Básicas de Saúde da Família inclua também o aspecto cognitivo, de maneira que a demência, a depressão e o comprometimento de memória sejam detectados e tratados precocemente, uma vez que o Ministério da Saúde recomenda que os profissionais de saúde reconheçam as demandas de saúde mental presentes nas diversas queixas relatadas pelos pacientes que chegam aos serviços de saúde da Atenção Básica, principalmente na Estratégia Saúde da Família ${ }^{62}$.

Os resultados obtidos neste estudo ressaltam a importância do acompanhamento da saúde dos idosos e do desenvolvimento e ações por parte dos profissionais de saúde nas Unidades Básicas de Saúde da Família, voltadas para o suporte desses idosos, que apresentam declínio cognitivo tais como a demência, a depressão e o comprometimento da memória, a fim de melhorar a qualidade de vida desta população. 


\section{Colaboradores}

NDS Sousa participou da revisão bibliográfica, da interpretação e discussão dos dados e redação do artigo. T Menezes participou da concepção e delineamento do estudo, da interpretação e discussão dos dados e da revisão do artigo. N Silva participou da análise estatística e interpretação dos dados. AA Paiva participou da revisão do artigo. MC Eulálio participou da revisão do artigo.

\section{Referências}

1. World Health Organization. Iron deficiency anaemia. Assentamant, prevention and control. A guide for programe managers. Geneva: WHO; 2001.

2. Gualandro SF, Hojaij NHSL, Jacob Filho W. Deficiência de ferro no idoso. Rev Bras Hematol Hemoter 2010; 32(Supl. 2):57-61.

3. Sgnaolin V, Engroff P, Ely LS, Schneider RH, Schwanke CHA, Gomes J, Morrone FB, de Carli GA. Hematological parameters and prevalence of anemia among free-living elderly in the south Brazil. Rev Bras Hematol Hemoter 2013; 35(2):115-118.

4. Silva CLA, Lima-Costa MF, Firmo JOA, Peixoto SV. Nível de hemoglobina entre idosos e sua associação com indicadores do estado nutricional e uso de serviços de saúde: Projeto Bambuí. Cad Saude Publica 2012; 28(11):2085-2094.

5. Barbosa DL, Arruda JKG, Diniz AS. Prevalência e caracterização da anemia em idoso do Programa de Saúde da Família. Rev Bras Hematol Hemoter 2006; 28(4):288-292.

6. Bang SM, Lee JO, Kim YJ, Lee KW, Lim S, Kim JH, Park YJ, Chin HJ, Kim KW, Jang HC, Lee JS. Anemia and activities of daily living in the Korean urban elderly population: results from the Korean longitudinal study on health and aging (KLOSHA). Ann Hematol 2013; 92(1):59-65.

7. Tettamanti M, Lucca U, Gandin F, Recchia A, Mosconi P, Apolone G, Nobili A, Tallone MV, Detoma P, Giacomin A, Clerico M, Tempia P, Savoia L, Fasolo G, Ponchio L, Della Porta MG, Riva E. Prevalence, incidence and types of mild anemia in the elderly: the "Health and Anemia" population-based study. Haematologica 2010; 95(11):1849-1856.

8. Mugisha JO, Baisley K, Asiki G, Seeley J, Kuper H. Prevalence, types, risk factors and clinical correlates of anaemia in older people in a rural Ugandan population. Plos One 2013; 8(10):783-794.

9. Gaskeel H, Derry S, Moore RA, McQuay HJ. Prevalence of anaemia in older persons: systematic review. BMC Geriatrics 2008; 8(1):1-8.

10. Santos IS, Scazufca M, Lotufo PA, Menezes PR, Benseñor IM. Anemia and dementia among the elderly: the São Paulo Ageing Health Study. Int Psychogeriatr 2012; 24(1):74-81.

11. Vanasse GJ, Berliner N. Anemia in elderly patients: an emerging problem for the $21^{\text {st }}$ century. Hematology Am Soc Hematol Educ Program 2010; 2010(1):271-275.

12. Ciquet MG. Anemia no idoso. Rev Bras Med 2010; 67(4):89-96.

13. Bosco RM, Assis EPS, Pinheiro RR, Queiroz LCV, Pereira LSM, Antunes CMS. Anemia and functional capacity in elderly brazilian hospitalized patients. Cad Saude Publica 2013; 29(7):1322-1332.

14. Price EA, Mehra R, Holmes TH, Schrier SL. Aging and erythropoiesis: current state of knowledge. Blood Cells Mol Dis 2008; 4(2):158-165.

15. Smith RE. The Clinical and economic burden of anemia. Am J Mang Care 2010; 16(Supl. 1):S59-66.

16. Nunes A. O envelhecimento populacional e as despesas do Sistema único de saúde. In: Camarano AA, organizador. Novos idosos brasileiros: muito além dos 60? Rio de Janeiro: Instituto de Pesquisa Econômica Aplicada; 2004. p. 427-450. 
17. Shah RC, Wilson RS, Tang Y, Dong X, Murray A, Bennetit DA. Relation of hemoglobin to level of cognitive function in older persons. Neuroepidemiol 2008; 32(1):40-46.

18. Peters R, Burch L, Warner J, Beckeet N, Poulter R, Bulpitt C. Haemoglobin, anaemia, dementia and cognitive decline in the elderly, a systematic review. BMC Geriatr 2008; 8(18):1-6.

19. Shah RC, Schneider JA, Leurgan S, Bennett DA. Association of lower hemoglobin level and neuropathology in comminity-dwelling older persons. J Alzheirms Dis 2012; 32(3):579-586.

20. Shah RC, Buchman AS, Wilson RS, Leurgans SE Bennett DA. Hemoglobin level in older persons and incident Alzheimer disease: prospective cohort analysis. Neurology 2011; 77(3):219-226.

21. Zakai NA, Katz R, Hirsch C, Shlipak MG, Chaves $\mathrm{PH}$, Newman AB, Cushman M. A prospective study of anemia status, hemoglobin concentration, and mortality in a elderly cohort. Arch Intern Med 2005; 165(19):2214-2220

22. Silva CLA, Lima-Costa MF, Firmo JOA, Peixoto SV. Anemia e nível de hemoglobina como fatores prognósticos da mortalidade entre idosos residentes na comunidade: evidências da coorte de idosos de Bambuí, Minas Gerais, Brasil. Cad Saude Publica 2013; 29(11):2241-2250.

23. Denny SD, Kuchibhatla MN, Cohen HJ. Impact of anemia on mortality, cognition, and function in community-dwelling elderly. Am J Med 2006; 119(4):927-934.

24. Patel KV. Epidemiology of anemia in older adults. Semin Hematol 2008; 45(4):210-217.

25. Tombaungh TN, McIntyre NI. The mini-mental state examination: a comprehensive review. J Am Geriatr Soc 1992; 40(9):922-935.

26. Ng TP, Feng L, Niti M, Yap KB. Albumin, haemoglobin, BMI and cognitive performance in older adults. Age Ageing 2008; 37(4):423-429.

27. Zilinski J, Zillmann R, Becker I, Benging T, Schulz RJ, Roehrig G. Prevalence of anemia among elderly impatients and its association with multidimensional loss of function. Ann Hematol 2014; 93(10):1645-1654.

28. Yesavage JA, Brink TL, Rose TL, Lum O, Huang V, Adey M. Development and validation of a geriatric depression screening scale: a preliminary report. $J$ Psychiatr Res 1983; 17(1):37-49.

29. Lucca U, Tettamanti M, Moscon P, Apolone G, Gandini F, Nobili A, Tallone MV, Detoma P, Giacomin A, Clerico M, Tempia P, Guala A, Fasolo G, Riva E. Association of mild anemia with cognitive, functional, mood and quality of life outcomes in the elderly: the "Health and Anemia" study. Plos One 2008; 3(4):1920-1928.

30. Thein M, Ershler WB, Art AS, Tecson J, Robinson BE, Rothstein G, Liede A, Gylys-Colwell I, Lu ZJ, Robbins $S$. Diminished quality of life and physical function in community-dowelling elderly with anemia. Medicine 2009; 88(2):107-114.

31. Crook TH, Feher EP, Larrabee GJ. Assessment of memory complaint in age-associated memory impairment: the MAC-Q. Int Psychogeriatr 1992; 4(2):165-176.

32. Chaves AS, Santos AM, Alves MTSSB, Salgado Filho N. Associação entre declínio cognitivo e qualidade de vida de idosos hipertensos. Rev Bras Geriatr Gerontol 2015; 18(3):545-556.
33. World Health Organization (WHO). Nutritional anemia: report of a WHO scientific group. Geneva: WHO; 1968.

34. Folstein MF, Folstein SE, McHugh PR. "Mini-Mental State": a practical method for grading the cognitive state of patients for the clinician. J Psych Res 1975; 12(3):189-198.

35. Almeida OP, Almeida SA. Short versions of the Geriatric Depression scale: a study of their validity for the diagnosis of a major depressive episode according to ICD-10 and DSM-IV. Int J Geriatr Psychiatry 1999; 14(10):858-865.

36. Gordon CC, Chumlea WC, Roche AF. Stature, recumbent length, and weight. In: Lohman TG, Roche AF, Martorell R, editors. Anthropometric standardization reference manual. Champaign: Human Kinetics Books; 1988. p. 3-8.

37. Organização Pan-Americana (OPAS). XXXVI Reunión del Comitê Asesor de Investigaciones em Salud - Encuestra Multicêntrica - Salud Bienestar y Envejecimiento (SABE) en América Latina e el Caribe - Informe preliminar. Washington: OPAS; 2002.

38. Rocha DS, Capanema FD, Netto MP, Franceschini SCC, Lamounir JA. Prevalência e fatores determinantes da anemia em crianças assistidas em creches. Rev Bras Epidemiol 2012; 15(3):675-684.

39. Vieira RCS, Ferreira HS. Prevalência de anemia em crianças brasileiras segundo diferentes cenários epidemiológicos. Rev Nutr 2010; 23(3):433-444.

40. Aragão FKS, Almeida AL, Nunes SFL. Prevalência e fatores associados à anemia em gestantes atendidas em uma maternidade pública no município de Imperatriz, Maranhão. J Manag Prim Health Care 2013; 4(3):183190.

41. Fujimori E, Sato APS, Szarfarc SC, Veiga GV, Oliveira VA, Colli C. Anemia em gestantes brasileiras antes e após fortificação das farinhas com ferro. Rev Saude Publica 2011; 45(6):1027-1035.

42. Corona LP, Duarte YAD, Lebrão ML. Prevalência de anemia e fatores associados em idosos: evidências do Estudo SABE. Rev Saude Publica 2014; 48(5):723-731.

43. Schraiber LB, Figueiredo WS, Gomes R, Couto MT, Pinheiro TF, Machin R, Silva GSN, Valença O. Necessidade de saúde e masculinidade: atenção primária no cuidado aos homens. Cad Saude Publica 2010; 26(5):961-970.

44. Figueiredo WS. Assistência à saúde dos homens: um desafio para os serviços de atenção primária. Cien Saude Colet 2005; 10(1):105-109.

45. Schaan MDA, Schwank CHA, Bauer M, Luz C, Cruz IM. Hematological and nutritional parameters in apparently healthy elderly individuals. Rev Bras Hematol Hemoter 2007; 29(2):136-143.

46. Ershler WB, Sheng S, Mckelvey J, Art AS, Denduluri N, Tecson J, Taub DD, Brant LJ, Ferrucci L, Longo DL. Serum erythropoietin and aging: a longitudinal analysis. J Am Geriatr Soc 2005; 53(8):1360-1365.

47. Ferruci L, Semba RD, Guaralnik JM, Ershler WB, Bandinelli S, Patel KV, Sun K, Woodman RC, Andrews NC, Cotter RJ, Ganz T, Nemeth E, Longo DL. Proinflammatory state, hepcidin and anemia in older persons. Blood 2010; 115(18):3810-3826 
48. Garcia ANM, Romani SAM, Lira PIC. Indicadores antropométricos na avaliação nutricional de idosos: um estudo comparativo. Rev Nutr 2007; 20(4):371-378.

49. Sousa VMC, Guariento ME. Avaliação do idoso desnutrido. Rev Bras Clin Med 2009; 7(1):46-49.

50. Sperotto FM, Spinelli RB. Avaliação nutricional em idosos independentes de uma instituição de longa permanência no município de Erechim-RS. Perspectiva 2010; 34(125):105-116.

51. Santos ACO, Machado MMO, Leite EM. Envelhecimento e alterações do estado nutricional. Rev Bras Geriat Gerontol 2010; 4(3):168-175.

52. Rolfes SR, Pina K, Whitney E. Understanding normal and clinical nutrition. $8^{\mathrm{a}}$ ed. Belmont: Wadsworth; 2009.

53. Deal JA, Carlson MC, Xue Q, Fried LP, Chaves PHM. Anemia and 9-year domain-specific cognitive decline in community-dwelling older women: The women's health and Aging Study II. J Am Geriatr Soc 2009; 57(9):1604-1611.

54. Parihar MS, Hemnani T. Alzheimer's disease pathogenesis and therapeutic interventions. J Clin Neurosci 2004; 11(5):456-467.

55. Sereniki A, Vital MABF. A doença de Alzheimer: aspectos fisiopatológicos e farmacológicos. Rev Psiquiatr 2008; 30 (Supl. 0):0-0.

56. Santos MD, Borges SM. Percepção da funcionalidade nas fases leve e moderada da doença de Alzheimer: visão do paciente e seu cuidador. Rev Bras Geriatr Gerontol 2015; 18(2):339-349.

57. Assaraf MI, Diaz Z, Liberman A, Miller WH, Arvanitabis Z, Bennett DA. Brain erythhropoietin receptor expressionin Alzheimer disease and mild cognitive impairment. J Neuropathol Exp Neurol 2007; 66(5):389398.

58. Ng TP, Feng L, Niti M, Yap KB. Albumin, haemoglobin, BMI and cognitive performance in older adults. Age Ageing 2008; 37(4):423-429.

59. Zilinski J, Zillmann R, Becker I, Benging T, Schulz RJ, Roehrig G. Prevalence of anemia among elderly impatients and its association with multidimensional loss of function. Ann Hematol 2014; 93(10):1645-1654.

60. Chalmers KA, Knuiman MW, Divitini ML, Bruce DG, Olynyk JK, Milward EA. Long-term mortality risks associated with mild anaemia in older persons: the Busselton Health Study. Age Ageing 2012; 41(6):759-764.

61. Brasil. Ministério da Saúde (MS). [acessado 2016 Mar 20]. Disponível em: http: http://dab.saude.gov.br/dab/ historico_cobertura_sf/historico_cobertura_sf_relatorio.php

62. Brasil. Ministério da Saúde (MS). Cadernos de Atenção Básica Saúde Mental. Brasília: MS; 2013.

Artigo apresentado em 17/12/2015

Aprovado em 18/06/2016

Versão final apresentado em 20/06/2016 